\section{Tuberous Sclerosis}

Carol L. Armstrong

Child and Adolescent Psychiatry and Behavioral

Sciences, The Children's Hospital of

Philadelphia, Philadelphia, PA, USA

\section{Definition}

Tuberous sclerosis occurring in the brain is a rare genetic disorder that can affect multiple critical organs, but when affecting the brain causes benign tumors to grow, and is associated with seizures, autism, mental retardation, behavioral problems, autism, and skin abnormalities, according to the National Institute of Neurological Disorders and Stroke. Cardiac lesions, renal lesions, pulmonary manifestations, and neurologic manifestations (epilepsy, infantile spasms, profound intellectual disability, subependymal giant-cell tumors causing obstructive hydrocephalus) are common. This disease cannot be cured and is managed through follow-up to monitor it and through treatment of symptoms. The common behavioral manifestations include increased rates of depression, anxiety, and aggressive outbursts (Pulsifer et al. 2007; deVries et al. 2007).

The tuberous sclerosis complex (TSC) is a multisystem, autosomal dominant disorder affecting children and adults resulting from mutations in either the TSC1 gene encoding hamartin or the TSC2 gene encoding tuberin (Crino et al. 2006).
The tubers are developmental abnormalities in the cerebral cortex made up of dysmorphic neurons, large astrocytes, and giant cells that developed in cortex that lost the normal six-layered structure (Crino et al. 2006).

Recent findings indicate that molecularly targeted treatments using inhibitors of the mammalian target of Rapamycin (mTOR), such as rapamycin and sirolimus, may improve the neurological and neuropsychiatric phenotype of tuberous sclerosis (deVries 2010). In addition to tuberous sclerosis, Rapamycin may also treat fragile $\mathrm{X}$ and neurofibromatosis type 1 syndromes, as well as disorders associated with phosphatase and tensin homo (PTEN) mutations, though these studies are ongoing.

\section{References and Reading}

Crino, P. B., Nathanson, K. L., \& Henske, E. P. (2006). The tuberous sclerosis complex. The New England Journal of Medicine, 355, 1345-1356.

deVries, P. J. (2010). Targeted treatments for cognitive and neurodevelopmental disorders in tuberous sclerosis complex. Neurotherapeutics, 7(3), 275-282.

deVries, P. J., Hunt, A., \& Bolton, P. F. (2007). The psychopathologies of children and adolescents with tuberous sclerosis complex (TSC): A postal survey of UK families. European Child and Adolescent Psychiatry, 16(1), 16-24.

Pulsifer, M. B., Winterkorn, E. B., \& Thiele, E. A. (2007). Psychological profile of adults with tuberous sclerosis complex. Epilepsy and Behavior, 10(3), 402-406. 